\title{
Correction to: Highlights of the annual scientific meeting of the 24th congress of the European Society of Musculoskeletal Radiology (ESSR) 2017
}

\author{
Carmelo Messina $^{1}$ • Luca Maria Sconfienza ${ }^{1,2}$ - Giuseppe Guglielmi ${ }^{3,4}$
}

Published online: 13 October 2017

(C) ISS 2017

\section{Correction to: Skeletal Radiol}

https://doi.org/10.1007/s00256-017-2774-7

The original version of this article unfortunately contained mistakes. The name and affiliations of Luca Maria Sconfienza are already corrected on this article.

The original article has been corrected.

The online version of the original article can be found at https://doi.org/ $10.1007 / \mathrm{s} 00256-017-2774-7$

Giuseppe Guglielmi

giuseppe.guglielmi@unifg.it

1 Unit of Diagnostic and Interventional Radiology, IRCCS Istituto Ortopedico Galeazzi, Via Galeazzi 4, 20161 Milan, Italy

2 Department of Biomedical Sciences for Health, Università degli Studi di Milano, Via Festa del Perdono 7, 20122 Milan, Italy

3 Department of Radiology, University of Foggia, Foggia, Italy

4 Department of Radiology, Scientific Institute "Casa Sollievo della Sofferenza” Hospital, San Giovanni Rotondo, Foggia, Italy 